\title{
Run-time Spatial Resource Management for Real-Time Applications on Heterogeneous MPSoCs
}

\author{
Timon D. ter Braak, Philip K.F. Hölzenspies, Jan Kuper, Johann L. Hurink, Gerard J.M. Smit \\ Department of Electrical Engineering, Mathematics and Computer Science \\ University of Twente, P.O. Box 217, 7500 AE Enschede, The Netherlands \\ t.d.terbraak@utwente.nl
}

\begin{abstract}
Design-time application mapping is limited to a predefined set of applications and a static platform. Resource management at run-time is required to handle future changes in the application set, and to provide some degree of fault tolerance, due to imperfect production processes and wear of materials. This paper concerns resource allocation at run-time, allowing multiple real-time applications to run simultaneously on a heterogeneous MPSoC. Low-complexity algorithms are required, in order to respond fast enough to unpredictable execution requests. We present a decomposition of this problem into four phases. The allocation of tasks to specific locations in the platform is the main contribution of this work. Experiments on a real platform show the feasibility of this approach, with execution times in tens of milliseconds for a single allocation attempt.
\end{abstract}

\section{INTRODUCTION}

Energy consumption has become a critical issue, both for high-end large-scale parallel systems, as well as for portable devices. For some application domains, specialized architectures deliver more performance per Watt than general purpose processors. Research has shown, that for such application domains, heterogeneous multi-processor systems (MPSoCs) can deliver higher performance at a given energy budget than homogeneous multi-core solutions [1].

Numerous tool-chains are developed for design-time usage to analyze, partition and program applications for MPSoCs [2]. However, at design-time, it is unknown when, and what combinations of applications are requested to be executed during the life-time of the system. Therefore, only a limited number of schedules can be derived at design-time, targeting a predefined set of applications [3].

MPSoCs require resource management at run-time to be able to circumvent hardware faults, to minimize the operational cost of system (e.g. energy), and to adapt to user demands, while not being restricted on beforehand in combinations of applications. Such a resource manager must run within a limited execution environment on the target platform, thus requiring low-complexity algorithms.

\section{A. Run-time Spatial Resource Management}

Currently, we assume that task migration is not possible without violation of any reasonable performance constraints. Therefore, the resource allocation problem we consider is

This research is conducted within the FP7 Cutting edge Reconfigurable ICs for Stream Processing (CRISP) project (ICT-215881) supported by the European Commission. a non-preemptive and non-clairvoyant scheduling problem. Heuristics may be used to both anticipate future events and to reduce the problem's search space, at the cost of the quality of the solutions. We present a decomposition of the problem into multiple phases, which leads to such a reduction of computational complexity compared to the original problem, that it makes resource allocation at run-time feasible.

At design-time, some application development effort is required, indicated with the partitioning phase in Fig. 1. An application is partitioned in multiple tasks [4], resulting in an application specification, which contains an annotated task graph and possibly some performance constraints. For each task, multiple implementations may be provided by different IP manufacturers, using multiple QoS levels, or targeting different memory types and I/O interfaces.

The application specification is used at run-time to find and allocate the required resources. We decompose this resource allocation problem in the following phases:

1) Binding: for each task of the application, an implementation is selected that is able to execute the task with low cost and sufficient performance. The required resources must be available somewhere in the platform.

2) Mapping: taking locality into account, specific resources are assigned to each task, such that the resource requirements of the implementations chosen in the binding phase, are fulfilled.

3) Routing: for pairs of tasks that need to communicate, communication links are established between the elements assigned to them in the mapping phase.

4) Validation: the performance constraints given in the application specification are validated against the performance provided by the execution layout derived from the previous phases.

As a result of these phases, an execution layout defines what specific resources are allocated to each task and communication channel in the application. Based on this, configuration software can configure the hardware accordingly and start the application, which we indicate with the bootstrapping phase.

\section{B. Outline}

The following section describes related work and our contribution. Section III explains our algorithm for the mapping phase, followed by some results we obtained with a prototype in Section IV. 


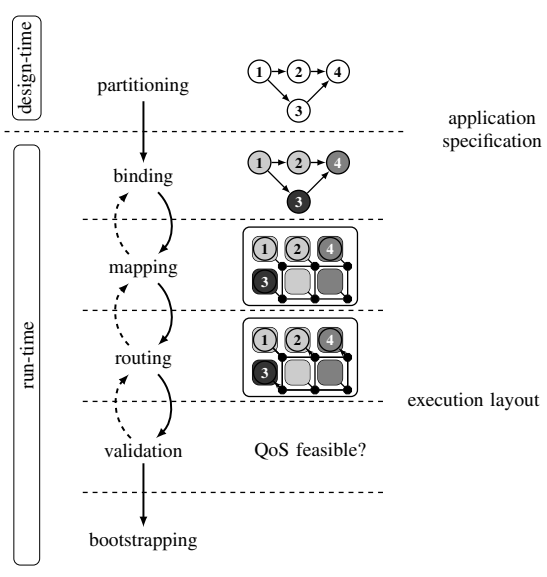

Fig. 1. Phases in run-time spatial resource management.

\section{Contribution And Related Work}

The resource allocation approach of [5] works directly on synchronous dataflow (SDF) graphs to provide timing guarantees, but its calculation time is too long for run-time usage; their strategy takes minutes on a high end processor. The communication load of tiles is taken into account, but the topological relations between tasks and elements in the platform are discarded. In [6], internal and external contention in communication streams is considered, but their region forming approach is targeted at homogeneous meshed platforms, and is not suitable for heterogeneous or irregular architectures. In [7], an architecture driven approach is used to map tasks first on virtual tiles, which are in turn clustered on elements connected to the same router. The distributed approach of [8] uses a static mapping algorithm inside its clusters. This approach requires hardware support for cluster management, while it poses more constraints on the size and structure of applications.

In this work, we propose a generic task mapping algorithm that works on a variety of platforms, using any cost function that can be defined for a platform. To this end, we have a notion of topology, but we do not make any assumption on the routing algorithm. Additionally, the calculation time of our approach makes it feasible for run-time usage, even when multiple iterations are required to improve the solution.

For the binding phase, we use the approach in [9], which selects for each task an implementation, ordered by the difference between the cheapest and second cheapest assignment, as in [10]. We use virtual channels to time-share communication resources in the platform [11]. The less complex breadthfirst search is used for routing, because it has no noticeable performance differences in terms of successful routes and energy consumption, compared to Dijkstra's algorithm [11]. For validation of the performance constraints of applications, we model the influence of the platform and the application specification as an SDF graph. We express latency constraints in the application as throughput constraints, as in [12]. With a state-space exploration of the SDF graph, presented in [5], [13], we calculate the throughput of the corresponding application, which determines whether any throughput or latency constraint is violated.

\section{MAPPING AlgORITHM}

In the mapping phase of the workflow illustrated by Fig. 1, we want to find specific locations to fulfill the resource requirements of the tasks $\mathcal{T}$ and channels $\mathcal{C}$ in an application $\mathcal{A}=\langle\mathcal{T}, \mathcal{C}\rangle$. A platform $\mathcal{P}=\langle\mathcal{E}, \mathcal{L}\rangle$ provides resources through the processing elements $\mathcal{E}$, which are connected with the links $\mathcal{L} \subseteq \mathcal{E} \times \mathcal{E}$. We propose an incremental mapping algorithm, in which we traverse both the task graph and the platform, while trying to match their topological structure. At various points, we allocate resources to a task from a subset of processing elements. A vector notation is used to denote the resources provided by elements, and the resources required by implementations [14]. Various mapping objectives may be defined, like minimal energy consumption, reducing resource fragmentation, wear leveling, or load balancing.

\section{A. Task Graph Traversal}

Our mapping heuristic uses divide-and-conquer to further break the mapping problem into sub-problems of variable size, depending on the density of the task graph. Especially in embedded systems, a subset of tasks in an application often has only one mapping option in the platform. This scenario occurs, for example, when the application requires specific interfaces for input and output data streams. While I/O operations may be generic in nature, locations may be fixed in the binding phase. Assuming such a scenario, let $\mathcal{T}_{0} \subseteq \mathcal{T}$ be the subset of tasks in application $\mathcal{A}$, that can be mapped to a single element $e \in \mathcal{E}_{0}$ only. Substantiating these relations results in a partial mapping $\mathcal{M}_{0}=\left\langle\mathcal{T}_{0}, \mathcal{E}_{0}\right\rangle$. Each sub-problem $i$ is then defined as a subset of tasks $\mathcal{T}_{i} \subseteq \mathcal{T}$, such that $\mathcal{T}_{i}$ is the $i^{t h}$ undirected neighborhood $N_{i}$ of $\mathcal{I}_{0}$. In other words:

1) We group the tasks in sets with equal distance to the origin $\operatorname{task}(\mathrm{s}) t \in \mathcal{T}_{0}$.

Maintaining the order of increasing distance $i$, each subproblem is then resolved by these two steps:

2) Search the platform for enough elements $\mathcal{E}_{i} \subseteq \mathcal{E}$ spatially close to $\mathcal{E}_{i-1}$, such that the resource requirements of the tasks in $\mathcal{T}_{i}$ are met.

3) Find a mapping $\mathcal{M}_{i}$ of the tasks in $\mathcal{T}_{i}$ to $\mathcal{E}_{i}$.

When $\mathcal{T}_{0}$ is initially empty, a starting point in the application has to be defined. We want to prevent situations where computational resources are isolated due to the lack of communication resources. We define external resource fragmentation as the percentage of pairs of adjacent elements of which only one element is used, over all pairs of adjacent elements in the platform. To reduce external fragmentation of processing elements, we select a task $t_{0}$ with a degree $d\left(t_{0}\right)$ that is the lowest in the task graph, indicated with $\delta(\mathcal{T})$. For this task, we search an element $e_{0} \in \mathcal{E}$ that is likely to become isolated later on, when it is not used now. Using $\left\langle t_{0}, e_{0}\right\rangle$ as $\mathcal{M}_{0}$, we continue with the three steps we just described. An example is given in Fig. 2; in the first step $i=0$, we take the dashed node as a starting point.

Task mapping requires us to reason about locality, and therefore a dependency exists between iterations of the algorithm. 

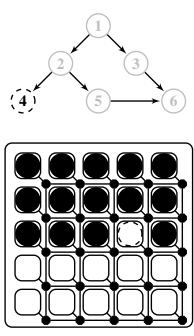

(a) $i=0$
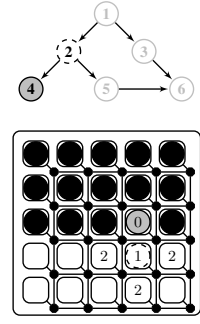

(b) $i=1$
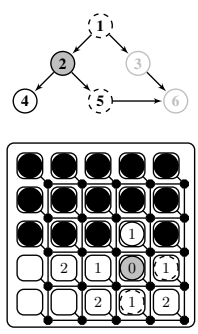

(c) $i=2$

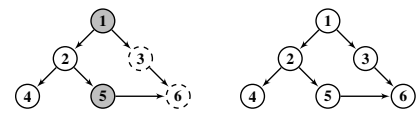

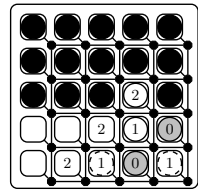

(d) $i=3$

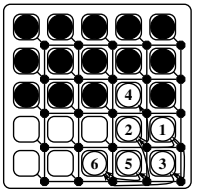

(e) finished
Fig. 2. Mapping state after each iteration in MapApplication, where gray nodes represent the partial mapping $\mathcal{M}_{i-1}$ and dashed nodes compose $\mathcal{M}_{i}$.

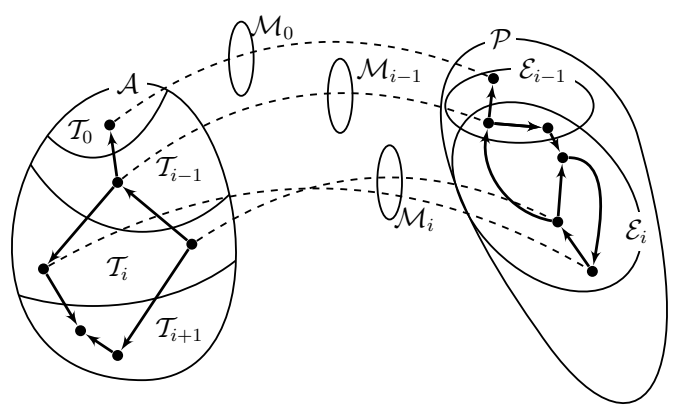

Fig. 3. For every subset $\mathcal{T}_{i}$ of tasks in application $\mathcal{A}$, a subset $\mathcal{E}_{i}$ of the elements in platform $\mathcal{P}$ is selected to form mapping $\mathcal{M}_{i}$.

This incremental mapping approach is illustrated in Fig. 3, and will be the subject of the following two sections.

\section{B. Searching for Elements}

While traversing the task graph, we have to find for every $\mathcal{T}_{i}$ a set of elements that provides enough resources to map all tasks in $\mathcal{T}_{i}$. An element $e$ is available for task $t$, writing $a v(e, t)$, if element $e$ can fulfill the resource requirements of the implementation for task $t$.

In every iteration, we start searching in the topological neighborhood of the elements that were allocated in the previous iteration. From the location of the elements $\mathcal{E}_{i-1}$, a breadth-first search (BFS) is started. When the partial mapping $\mathcal{M}_{i-1}$ contains more than one element, we start this search at multiple locations (see Fig. 2d). In the BFS, we try to match the communication infrastructure of the platform to the structure of the task graph, by taking the direction of communication channels between tasks into account. In this search, we keep track of the distance between a newly discovered element and the origins of the BFS, to estimate the cost of the communication routes.
Due to the multiple optimization objectives in the mapping phase, we do not stop searching for elements if we found exactly enough elements. This would facilitate only the minimal communication distance objective, and would make, for example, the resource fragmentation objective less effective. Thus, once we have discovered enough elements in the platform to map the tasks in $\mathcal{T}_{i}$, a single additional search step is performed. This results in a set of candidate elements that is likely to contain more elements than the tasks in $\mathcal{T}_{i}$ require. Based on the ratio between computation and communication cost, the local search can be extended to gather even more elements.

Up to this point, we described a search method that breaks the larger mapping problem into smaller sub-problems. We still have sets of tasks and elements, but they are much smaller than the entire application or platform. For each task $t \in \mathcal{T}_{i}$, an element $e \in \mathcal{E}_{i}$ has to be selected. Due to resource constraints, not all solutions are feasible; additionally, we want a solution that respects our optimization criteria. The following section describes this assignment problem.

\section{Assigning Tasks to Elements}

The sub-problems we have to solve, are instances of the generalized assignment problem (GAP). A GAP describes a problem where a number of items have to be placed in a number of bins. When the GAP has only one bin, the problem reduces to a knapsack problem. In our case, we consider elements to be bins with the resource capacities being the size of the bin. The tasks are the items that have to be placed in those bins, such that the resource requirements are met, and a minimum cost is achieved. In [15], an efficient algorithm for GAP is presented, with a time complexity of $O(\mathcal{E} \cdot k(\mathcal{T})+\mathcal{E} \cdot \mathcal{T})$, where $k(\mathcal{T})$ indicates the time complexity of a subroutine that solves knapsack problems. This algorithm guarantees a $(1+\alpha)$-approximation solution, where $\alpha$ is the approximation ratio of the knapsack subroutine. These characteristics state that both the quality and time complexity of this approach mostly depend on the knapsack solver.

Adopting the approach of [15], we iterate over the elements $\mathcal{E}_{i}$ that were discovered in MapApplication. For every $e \in \mathcal{E}_{i}$, we calculate for each $t \in \mathcal{T}_{i}$ the cost of mapping task $t$ to element $e$. We put these values in a vector $c_{2}$ of length $\left|\mathcal{T}_{i}\right|$. Another vector $c_{1}$ contains the cost of the best known mappings in $\mathcal{M}_{i}$, initially set to very large values. We pass both vectors to a knapsack routine that selects for that single element a subset of tasks with a minimal total cost. When an element $e$ picks a task $t$, the cost of that combination is stored as $c_{1}(t)$. Any subsequent evaluations for $e^{\prime} \in \mathcal{E}_{i}$ consider the cost reduction over that combination. Thus, we only consider remapping a task $t$, if the cost reduction $c_{1}(t)-c_{2}(t)$ is positive. Most of the time, picking a yet unmapped task is more beneficial than remapping a task to another element.

The procedure SolvegAP gathers all the partial mappings, and returns them to caller MapApplication. If insufficient elements were supplied to map every task, then 


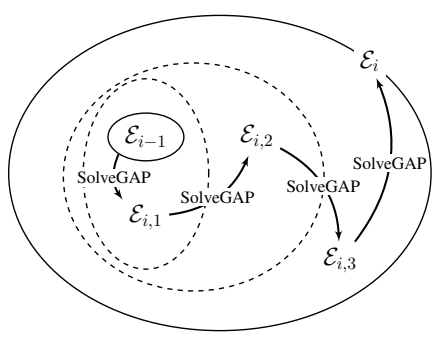

Fig. 4. Starting from the elements of the previous iteration $\mathcal{E}_{i-1}$, the set of candidate elements $\mathcal{E}_{i}$ is expanded, until a feasible mapping $\mathcal{M}_{i}$ is found.

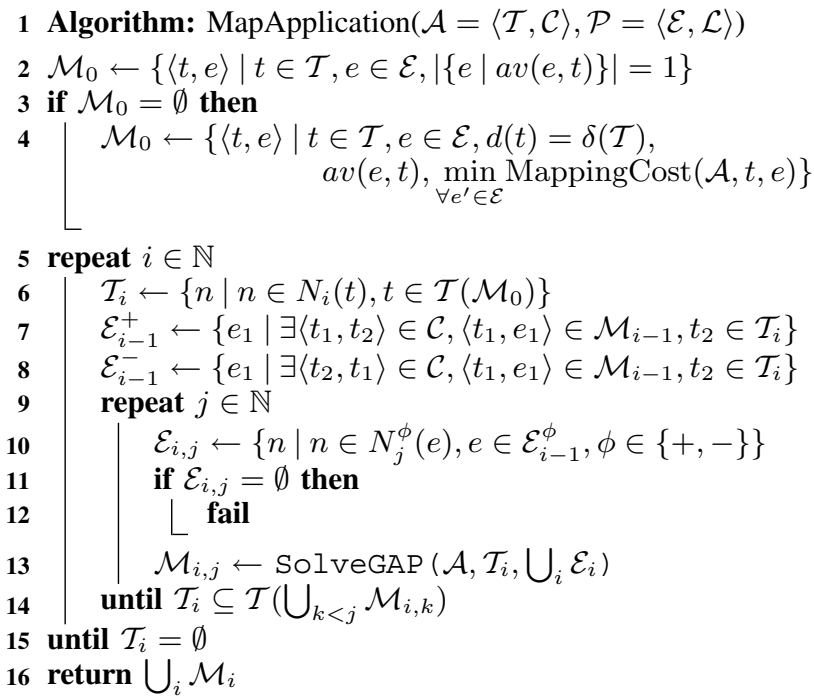

Fig. 5. Algorithm MapApplication

MapApplication will invoke SolveGAP again, but with a larger set of elements. Fig. 4 shows the growth of the set of elements $\mathcal{E}_{i}$, until SolveGAP manages to map all tasks in $\mathcal{T}_{i}$. During this process, the set of tasks remains unchanged, allowing us to reuse the mappings and their associated cost, as determined in the previous invocation. Note that when the cost function depends on the state of the partial mapping $\mathcal{M}_{i}$, it must be re-evaluated every time $\mathcal{M}_{i}$ changes, resulting in an increased complexity. Our knapsack implementation has a time complexity $O\left(\mathcal{T}^{2}\right)$.

The algorithm we propose is listed in Fig. 5. An example of the mapping process is given in Fig. 2. The actual result depends mostly on the definition of the cost function. While it is hard to define a good cost function, it also provides the flexibility to switch between optimization criteria. In our case, we assume that every task has to be mapped to avoid rejection of the application. The next section defines the cost function we use to make the actual decisions.

\section{Mapping cost function}

To evaluate the cost of mapping a task $t$ to an element $e$, we first look at the total communication distance involved with candidate element $e$. A sparse distance matrix is built while searching the platform for elements. If a required distance lookup fails, a relative high penalty is given to $e$, because then we assume a large communication distance between element $e$

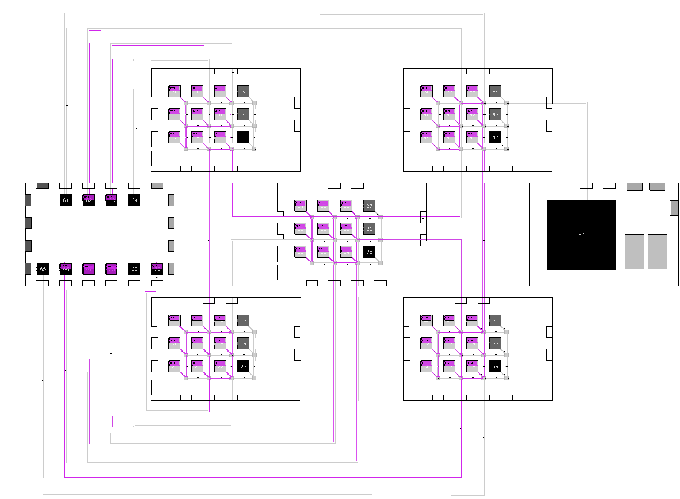

Fig. 6. The CRISP platform, composed by an ARM processor (right), an FPGA (left), and 5 packages of 9 DSPs, 2 memories and 1 hardware test unit.

and one of the communication peers of task $t \in \mathcal{T}_{i-1}$. For yet unmapped tasks $\mathcal{T}_{i+1}$, the distance is inherently unknown, and therefore left out of the equation.

The other mapping objective we consider is external resource fragmentation. An element $e$ receives decreasing bonuses for neighbor elements that retain communication peers of $t$, tasks from the same application $\mathcal{A}$, or tasks from other applications. Additionally, the connectivity of an element $e$ is taken into account as well; elements on the borders of chips are thus more favorable to use. The ratio between these two objectives is given by weight parameters, which can steer the resource manager towards minimal internal or external contention [6].

\section{E. Implementation}

A prototype resource manager named "Kairos" has been developed, containing the work-flow of Fig. 1. This prototype is integrated in a Linux $2.6 .28 \mathrm{kernel}$, running on a $200 \mathrm{MHz}$ ARM926Ej-S processor, using about $16 \mathrm{MB}$ of SDRAM. We specified a binary format for applications, that allows integration of the task graph, specification, and task implementations. As Linux supports multiple binary formats for executables, a new binary handler can distinguish MPSoC applications from operating system tools. In this paper, we illustrate our algorithms with the platform in Fig. 6; this platform is under development in the CRISP project [16].

\section{EXPERIMENTAL RESUlTS}

We use an in-house developed application generator, which is similar to TGFF [17], to generate six synthetic datasets. In this tool, the structure of an application can be specified with a number of input, internal, and output tasks. Also the maximum in-degree and out-degree of tasks gives direction to the generated communication structure. For each task, we generate a number of task implementations, annotated with bounded random resource requirements.

We generate applications that are either computational intensive or communication oriented. Tasks in the first set use between $70 \%$ and $100 \%$ of the element's resources, and tasks in communication oriented applications use between $10 \%$ and $70 \%$. This allows for communication oriented applications to 
TABLE I

Dataset Characteristics and Failure Percentage Per Phase.

\begin{tabular}{lccrlr}
\hline \multicolumn{2}{c}{ Dataset } & \multicolumn{4}{c}{ Failure Distribution } \\
\multicolumn{2}{c}{ Characteristics } & \#App & Binding & Mapping & Routing \\
\hline Communication & Small & 97 & $0.65 \%$ & $0.40 \%$ & $98.95 \%$ \\
Communication & Medium & 57 & $13.50 \%$ & $1.82 \%$ & $84.68 \%$ \\
Communication & Large & 22 & $3.45 \%$ & $0.00 \%$ & $96.55 \%$ \\
Computation & Small & 99 & $95.34 \%$ & $0.02 \%$ & $4.66 \%$ \\
Computation & Medium & 94 & $87.26 \%$ & $0.02 \%$ & $12.72 \%$ \\
Computation & Large & 96 & $61.64 \%$ & $0.31 \%$ & $38.05 \%$ \\
\hline
\end{tabular}

time-share elements, eventually resulting in communication bottlenecks. Within this characteristic, we categorize applications based on their size, namely small $(<5$ tasks), medium (6-10 tasks) and large (11-16 tasks) applications.

Tab. I shows the six datasets, each initially containing 100 applications. To filter out any extraneous samples, we remove applications from the dataset that cannot be mapped to an empty platform. For each dataset, we generate 30 random sequences of the remaining applications. We benchmark the platform with each dataset, by sequentially adding the applications to the platform. Between sequences the platform is emptied. Relatively early in the sequence, most platform resources are allocated, resulting in rejection of the remaining applications. Tab. I shows per phase the percentage of rejected applications as a function of all failing applications in a dataset. Because it is difficult to generate reasonable performance constraints automatically, we do not reject applications in the validation phase. The results show that a lack of communication resources generally causes the rejection of a communication oriented application. Computation intensive applications are mostly rejected in the binding phase. In the dataset with large, computation intensive applications, the communication resource requirements also become significant, resulting in more failures in the routing phase.

For successful resource allocation attempts, the average execution time of each phase in the resource manager is plotted in Fig. 7. This approach scales quite well for realistic application sizes, except for the validation phase. Throughput analysis requires a simulation of the corresponding dataflow graph; the length of the simulation only partly depends on the size of the application. Future work refers to another approach to handle the validation problem.

To qualify the mapping cost function, we investigate the influence of the mapping objectives. We optimize towards communication minimization, fragmentation reduction, and a combination of both objectives. Also, we disable the cost function, indicated with "None". The resulting execution layouts then depends on the communication minimization that is inherent to the resulting first-fit search method.

Fig. 8 shows the allocated number of hops per communication channel. After the $15^{\text {th }}$ application, the mapping success rate drops below $20 \%$. The applications that are still admitted, are allocated less communication resources compared to applications earlier in the sequence. This indicates that an application is only admitted to an almost saturated platform,

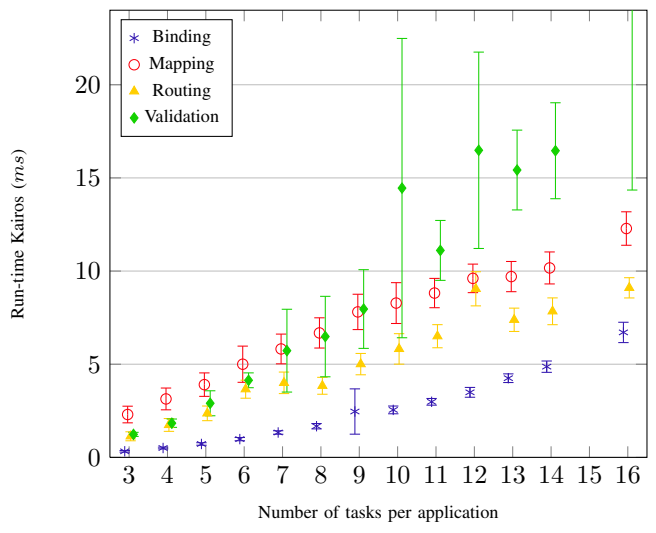

Fig. 7. Runtimes of Kairos for the applications in the synthetic datasets.

if an area with adjacent elements is still available.

Fig. 9 shows the external resource fragmentation of the elements in the platform, in relation to the progression of the application sequence. We see that the fragmentation converges to $30 \%$ and the mapping success rate converges to $10 \%$. Although it is not an absolute measure, it gives an idea of the required resource overhead (in terms of elements) in the platform. Compared to a fully meshed platform, the CRISP architecture is less connected. Aiming at fragmentation reduction (Fig. 9) increases the average communication distance (Fig. 8), resulting in a lower mapping success rate.

\section{A. Case Study: a Beamforming Application}

Fig. 6 shows a beamforming application developed for the CRISP platform. Containing 53 tasks in a tree-like structure, this application requires all 45 DSPs available in the platform, and can thus be considered to be a difficult mapping problem. Allocating resources for this application takes $70.4 \mathrm{~ms}$ for binding, $21.7 \mathrm{~ms}$ for mapping, $7.4 \mathrm{~ms}$ for routing, and $20.6 \mathrm{~ms}$ for validation. Although binding is fast for small applications, here it is actually the bottleneck. Furthermore, we see that the mapping algorithm scales quite well.

To analyze the influence of the mapping objectives, we vary the weights used in the cost function. Fig. 10 shows that only specific ratio between the fragmentation and communication objective results in admission of the application. Each contiguous area relates to a different mapping. Disabling either one of the objectives never gives a successful result.

\section{Discussion And Future Work}

In our decomposition of the spatial resource allocation problem, the mapping and validation phase are most complex. Our experiments show, that the mapping algorithm presented in this paper scales well with similar execution times compared to the other phases. The total execution time required for a single resource allocation attempt takes tens of milliseconds.

We showed that the resource manager can be steered by altering the cost function. In future research, we compare these results with an ILP formulation to determine the quality of the resource allocations. This is difficult, because we take overall objectives of the system into account, opposed to optimizing solutions of single applications. 


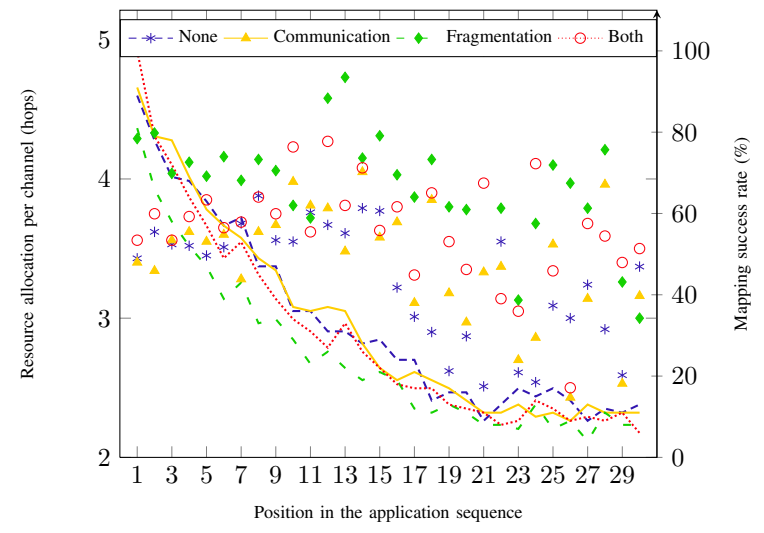

Fig. 8. Average communication resources allocated per channel.

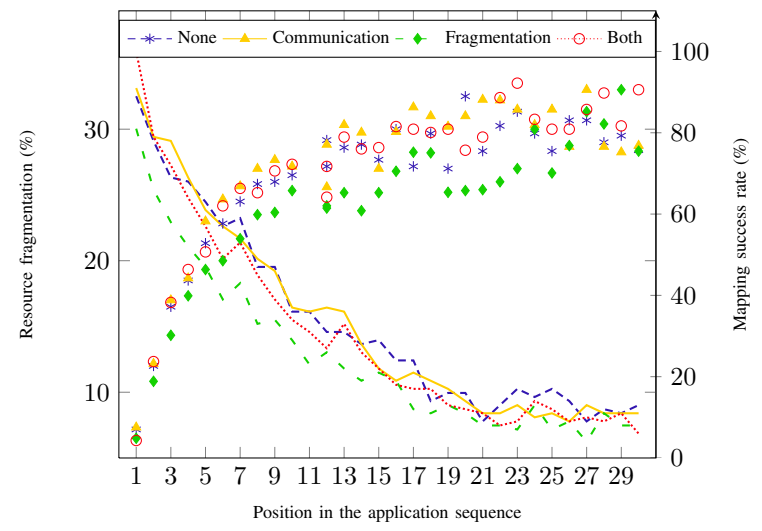

Fig. 9. External fragmentation of platform resources, averaged over all datasets, using various optimization criteria.

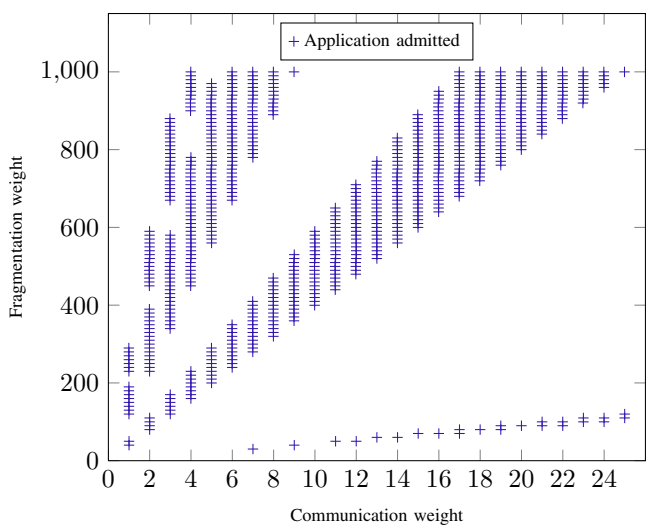

Fig. 10. Admission of a beamforming application with various mapping parameters. Every point in $[0,1, \ldots, 25] \times[0,10, . ., 1000]$ is sampled.

Future work also includes improving the validation method, which clearly becomes problematic when the complexity of the task graph increases. Besides the long calculation time, it is also difficult to cheaply generate feedback information. Using the work of [18], the complexity of the throughput analysis may be moved to design-time, making the validation approach a lot faster. The validation phase as a post-processing step can then be turned into a set of linear expressions that can be checked in parallel with the other phases.

\section{REFERENCES}

[1] R. Kumar, K. I. Farkas, P. Ranganathan, and D. M. Tullsen, "SingleISA heterogeneous multi-core architectures: The potential for processor power reduction," in Proc. of the 36th annual IEEE/ACM International Symposium on Microarchitecture. Washington, DC, USA: IEEE Computer Society, 2003, pp. 81-92.

[2] G. Martin, "Overview of the MPSoC design challenge," in DAC '06: Proceedings of the 43rd annual Design Automation Conference. New York, NY, USA: ACM, 2006, pp. 274-279.

[3] A. Hansson, M. Coenen, and K. Goossens, "Undisrupted quality-ofservice during reconfiguration of multiple applications in networks on chip," in DATE '07: Proc. of the conference on Design, automation and test in Europe. San Jose, CA, USA: EDA Cons., 2007, pp. 954-959.

[4] C.-Y. Yang, J.-J. Chen, T.-W. Kuo, and L. Thiele, "An approximation scheme for energy-efficient scheduling of real-time tasks in heterogeneous multiprocessor systems," in DATE '09: Proc. of the conference on Design, automation and test in Europe. New York, NY, USA: ACM, 2009, pp. 694-699.

[5] S. Stuijk, T. Basten, M. C. W. Geilen, and H. Corporaal, "Multiprocessor resource allocation for throughput-constrained synchronous dataflow graphs," in DAC '07: Proc. of the 44th annual Design Automation Conference. New York, NY, USA: ACM, 2007, pp. 777-782.

[6] C.-L. Chou and R. Marculescu, "User-aware dynamic task allocation in networks-on-chip," in DATE '08: Proc. of the conference on Design, automation and test in Europe. New York, NY, USA: ACM, 2008, pp. 1232-1237.

[7] O. Moreira, J. J.-D. Mol, and M. J. G. Bekooij, "Online resource management in a multiprocessor with a network-on-chip,' in SAC '07: Proc. of the 2007 ACM symposium on Applied computing. New York, NY, USA: ACM, 2007, pp. 1557-1564.

[8] M. A. A. Faruque, R. Krist, and J. Henkel, "ADAM: run-time agentbased distributed application mapping for on-chip communication," in DAC '08: Proc. of the 45th annual conference on Design automation. New York, NY, USA: ACM, 2008, pp. 760-765.

[9] P. K. F. Hölzenspies, J. L. Hurink, J. Kuper, and G. J. M. Smit, "Runtime spatial mapping of streaming applications to a heterogeneous multiprocessor system-on-chip," in DATE '08: Proc. of the conference on Design, automation and test in Europe, Mar. 2008, pp. 212-217.

[10] S. Martello and P. Toth, Knapsack problems: algorithms and computer implementations. New York, NY, USA: John Wiley \& Sons, Inc., 1990.

[11] N. Kavaldjiev, G. J. M. Smit, P. T. Wolkotte, and P. G. Jansen, "Providing QoS guarantees in a NoC by virtual channel reservation," in $A R C, 2006$, pp. 299-310.

[12] O. M. Moreira and M. J. G. Bekooij, "Self-timed scheduling analysis for real-time applications," in EURASIP Journal on Advances in Signal Processing, vol. 2007. Hindawi Publishing Corp., Apr. 2007, pp. 24-37.

[13] A. H. Ghamarian, M. C. W. Geilen, S. Stuijk, T. Basten, A. J. M. Moonen, M. J. G. Bekooij, B. D. Theelen, and M. R. Mousavi, "Throughput analysis of synchronous data flow graphs," Application of Concurrency to System Design, 2006. ACSD 2006. Sixth International Conference on, pp. 25-36, Jun. 2006.

[14] P. K. F. Hölzenspies, J. Kuper, G. J. M. Smit, and J. L. Hurink, "Demonstration of run-time spatial mapping of streaming applications to a heterogeneous multi-processor system-on-chip (MPSoC)," in Dagstuhl Seminar Proceedings 07101, Dagstuhl Wadern, Germany, B. R. H. M. Haverkort, J. P. Katoen, and L. Thiele, Eds., vol. 07101. Dagstuhl, Germany: Internationales Begegnungs- und Forschungszentrum für Informatik (IBFI), Oct. 2007.

[15] R. Cohen, L. Katzir, and D. Raz, "An efficient approximation for the generalized assignment problem," Inf. Process. Lett., vol. 100, no. 4, pp. 162-166, 2006.

[16] Recore Systems BV. (2008, Feb.) CRISP - cutting edge reconfigurable ICs for stream processing. FP7-ICT-215881. [Online]. Available: http://www.crisp-project.eu

[17] R. P. Dick, D. L. Rhodes, and W. Wolf, "TGFF: task graphs for free," in CODES/CASHE '98: Proc. of the 6th international workshop on Hardware/software codesign. Washington, DC, USA: IEEE Computer Society, 1998, pp. 97-101.

[18] A. H. Ghamarian, M. C. W. Geilen, T. Basten, and S. Stuijk, "Parametric throughput analysis of synchronous data flow graphs," in DATE '08: Proc. of the conference on Design, automation and test in Europe. New York, NY, USA: ACM, 2008, pp. 116-121. 